Canad. Math. Bull. Vol. 22 (3), 1979

\title{
COHERENT OVERRINGS
}

BY

IRA J. PAPICK

1. Introduction. In the study of particular categories of integral domains, it has proved useful to know which overrings of the domains of interest lie within the category, and indeed whether all such overrings do. (Recall: an overring of $R$ is a ring $T$ with $R \subseteq T \subseteq$ quotient field of $R$.) Two classes of domains classically studied in this setting are Prüfer domains and one-dimensional Noetherian domains. Since both of these classes are contained in the category of coherent domains, it is natural to investigate this category in this setting. (Recall: a ring is coherent if each finitely generated ideal is finitely presented.)

A preliminary version of this paper showed that Prüfer domains are exactly the integrally closed domains with all overrings coherent. The intent of this paper is to further develop this idea and to relate it to the definitive papers of Davis [4] and of Wadsworth [19] concerning particular "pairs" of integral domains. Our main results are accomplished by relating the "normal pairs" of Davis to our "coherent pairs" (definitions in §2).

Throughout this paper, all rings considered will be domains, i.e., commutative integral domains with identity, and unless otherwise specified, we shall always assume these domains are not fields.

Any unexplained terminology is standard as in [2] or [9].

2. Coherent pairs. By a pair $(R, T)$ we understand that $R \subseteq T$ is an extension of domains; a ring $S$ such that $R \subseteq S \subseteq T$ is called an intermediate domain of the pair $(R, T)$. The pair $(R, T)$ is called: coherent (resp., Noetherian) if each intermediate domain is coherent (resp., Noetherian [19]); normal if each intermediate domain is integrally closed in $T$ [4]; INC if $R \subseteq S$ is an INCextension for each intermediate domain $S$. (Recall [9]: a ring extension $R \subseteq T$ satisfies INC if given any prime ideals $P \subsetneq Q$ in $T, P \cap R \neq Q \cap R$.)

Guided by the treatment of normal pairs in [4] and of Noetherian pairs in [19], we are led to formulate three "natural" problems concerning coherent pairs:

PRoblem I. Determine those domains having all overrings coherent.

Problem II. Given a coherent domain $R$, determine all domains $T$ such that $(R, T)$ is coherent (and in particular, all such overrings $T$ ).

Received by the editors April 22, 1976 and, in revised forms, September 12, 1977 and August 3, 1978 . 
Problem III. Given a coherent domain $T$, determine all domains $R$ such that $(R, T)$ is coherent (and in particular determine all such $R$ so that $T$ is an overring of $R$ ).

We have had no success on III, whereas our progress on I (§3) and II (Corollary 8) is quite substantial.

We now collect a number of elementary facts concerning the pairs defined above. Hereafter we shall treat these as standard facts to be used without explicit comment or reference.

ElEMENTARY PROPERTIES OF PAIRS. Noetherian pairs are coherent. One easily shows: if $(R, T)$ is INC, then $T$ is algebraic over $R$; if $(R, T)$ is normal, then $T$ is an overring of $R$. It is straightforward to prove the equivalence of:

(1) $(R, T)$ is INC;

(2) $\left(S_{1}, S_{2}\right)$ is INC for all intermediate domains $S_{1} \subseteq S_{2}$;

(3) $\left(R^{\prime}, T\right)$ is INC for $R^{\prime}$ the integral closure of $R$ in $T$;

(4) $\left(R_{P}, T_{R \backslash P}\right)$ is INC for all prime (resp., maximal) ideals $P$ of $R$;

(5) $\left(S^{-1} R, S^{-1} T\right)$ is INC for each multiplicative system $S$ in $R$;

(6) $R \subseteq R[t]$ is an INC-extension for each $t \in T$.

One can recall or easily prove that in the case of the corresponding statements for normal pairs: (1) $\Leftrightarrow(2) \Leftrightarrow(4) \Leftrightarrow(5)$, while in the case of the corresponding statements for coherent pairs: $(1) \Leftrightarrow(2)$; and (1) $\Rightarrow(4),(5)$ [8, Corollary 3.1].

Proposition 1. If $(R, T)$ is coherent, then $(R, T)$ is INC.

Proof. If $(R, T)$ is not INC, then there is a $t \in T$ and prime ideals $P \digamma_{F} Q$ in $R[t]$ such that $P \cap R=Q \cap R \neq 0$. We may assume $R$ is local with maximal ideal $M=P \cap R$. Let $S$ be the integral closure of $R+P$ in $R[t]$. It is clear that $P$ is a maximal ideal of $S, t \notin S$, and $P=\left(S:_{S} t\right)$. Thus, as $S$ is coherent, $P$ is finitely generated. Hence since $t P \subseteq P, t$ is integral over $S$, a contradiction.

Proposition 2. $(R, T)$ is normal if and only if $(R, T)$ is INC with $R$ integrally closed in $T$.

Proof. We may assume $R$ is local with maximal ideal $M$. The "only if" part follows from [4, Proposition 2]. As for the other direction, suppose $R$ is integrally closed in $T$, but that $(R, T)$ is not normal. Then there is a $t \in T \backslash R$ with $t^{-1} \notin R[4$ Proposition 1]. So for $x$ an indeterminate, $M R[x] \supseteq$ $\operatorname{ker}(R[x] \rightarrow R[t]$ ) (see proof of [9, Theorem 67]), and hence $M R[t]$ is a non-maximal prime ideal of $R[t]$. Whence, $R \subseteq R[t]$ violates INC, which completes the proof.

THEOREM 3. $(R, T)$ is coherent with $R$ integrally closed in $T$ if and only if $(R, T)$ is normal with $R$ coherent. 
Proof. To conclude that $(R, T)$ is a normal pair with $R$ coherent we need only appeal to Propositions 1 and 2 . As for the converse, let $I$ be a finitely generated ideal of an intermediate domain $S$. Say $I=\sum S\left(a_{i} / b\right),\left(a_{i}, b \in R\right)$ and let $J=\sum R a_{i}$. Then $I \simeq J S$, and since $S$ is $R$-flat [4, Theorem 3], $J \bigotimes_{R} S \simeq J S$. Hence, $I$ is a finitely presented ideal of $S$, since $J$ is a finitely presented ideal of $R$. (It is worthwhile to mention that this direction also follows from the more general fact that coherence ascends flat epimorphisms [7, Proposition 2.2].)

COROLlaRY 4. ("Factorization of coherent pairs") Let $R^{\prime}$ denote the integral closure of $R$ in $T$. Then, $(R, T)$ is coherent if and only if $\left(R, R^{\prime}\right)$ and $\left(R^{\prime}, T\right)$ are coherent.

Proof. It is enough to prove the "if" direction. Since $\left(R^{\prime}, T\right)$ is INC (Proposition 1), we know that $(R, T)$ is INC. Given $R \subseteq S \subseteq T$, let $R^{\prime \prime}$ be the integral closure of $R$ in $S$. But $\left(R^{\prime \prime}, S\right)$ is normal (Proposition 2), and so $S$ is coherent (Theorem 3).

Remark 5. ("Factorization of Noetherian pairs") We first comment that the Noetherian analogue of Theorem 3 is valid and follows from the fact that Noetherian rings ascend flat epimorphisms [17, Corollary of Theorem 3]. Hence, by aping the proof of Corollary 4 we may conclude that $(R, T)$ is a Noetherian pair if and only if $\left(R, R^{\prime}\right)$ and $\left(R^{\prime}, T\right)$ are Noetherian pairs. (See $[19, \S 3]$ for a more general discussion of factorization of Noetherian pairs.)

Recall from [4] that a prime ideal $P$ of $R$ is trivial if there is no proper extension $R_{P} \subseteq T$ such that $\left(R_{P}, T\right)$ is normal. Note that if $h t(P)=1$, then $P$ is trivial if and only if $R_{P}$ is not a valuation domain ([4, Theorem 1]). Now [4, Theorem 2] says: $(R, T)$ is normal if and only if $T \subseteq \bigcap\left\{R_{P}: P\right.$ trivial $\}$; and moreover, in that event, $T=\bigcap\left\{R_{P}: T \subseteq R_{P}\right\}$. This fact together with Corollary 4 "factor" Problem II (and also I) into two quite distinct problems, reducing the problem, in effect, to a question of integral extensions.

TheOREM 6. Let $R^{\prime}$ be the integral closure of $R$ in $T$. Then:

(a) $(R, T)$ is coherent if and only if $\left(R, R^{\prime}\right)$ is coherent and $T \subseteq \bigcap\left\{R_{P}^{\prime}: P\right.$ trivial $\}$; and moreover, in this event, $T=\bigcap\left\{R_{P}^{\prime}: T \subseteq R_{P}^{\prime}\right\}$.

(b) (cf. [4], [5], [10], [18], [19]) Suppose $T \subseteq \bigcap\left\{R_{P}^{\prime}: h t(P) \neq 1\right\}$. Then, $(R, T)$ is coherent if and only if $\left(R, R^{\prime}\right)$ is coherent; and moreover, in this event, $T=\bigcap\left\{R_{M}^{\prime}: T \subseteq R_{M}^{\prime}, M\right.$ maximal or zero $\}$.

(c) Suppose $\operatorname{dim}(R)=1$. Then $(R, T)$ is coherent if and only if $T$ is algebraic over $R$ and $\left(R, R^{\prime}\right)$ is coherent.

Proof. By Corollary 4 we may assume that $R=R^{\prime}$ and $R$ is coherent, and by Theorem 3 we may focus on normality instead of coherence. Hence, (a) follows immediately from the above remarks (i.e., from [4, Theorem 2]). Note that (c) is clearly a special case of (b). By part (a) and the above remarks, to prove (b) it 
suffices to show that $T \subseteq R_{P}$ whenever $h t(P)=1$ and $R_{P}$ is not a valuation domain. Let $P$ be any height 1 prime such that $T \not \subset R_{P}$. Thus $R_{P} \subsetneq T_{R \backslash P}$ is a proper extension with $R_{P}$ integrally closed in $T_{R \backslash P}$. Let $S$ be an intermediate domain of the pair $\left(R_{P}, T_{R \backslash P}\right)$ which is not a field. Observe that the hypotheses of [11, Theorem 2] apply to the extension $R_{P} \subseteq S$, and so $R_{P}=S$. Therefore $R_{P}$ is a valuation domain.

Although Theorem 6 provides a "solution" to Problem II, it is by no means completely satisfactory. However, what the theorem does do is to split the problem into the following two parts, and hence helps bring into focus the underlying difficulties.

(i) Given an arbitrary coherent domain $R$, determine all integral extensions $R \subseteq R^{\prime}$ such that $\left(R, R^{\prime}\right)$ is coherent.

(ii) Given an arbitrary coherent domain $R^{\prime}$, determine all trivial prime ideals of $R^{\prime}$.

By specializing to the case of $R^{\prime}$ integrally closed, the following proposition resolves (ii).

Proposition 7. (cf. Proof of [4, Proposition 6]) Let $R$ be local, integrally closed, and $T$ an overring of $R$. If $(R, T)$ is a non-trivial coherent pair, then $R$ is a valuation domain.

Proof. By Theorem 3, $(R, T)$ is a non-trivial normal pair, and so $T=R_{N}$, $R / N$ is a valuation domain and $N=N R_{N}$ for some non-maximal prime ideal $N$ of $R$ [4, Proposition 2]. Thus it suffices to prove that $R_{N}$ is a valuation domain ("Composition of valuations"). Let $a \notin N$ be a non-unit of $R$, and let $P$ be a prime ideal minimal over $R a$. By [4, Proposition 2], $N \subseteq P$. Hence it suffices to show that $R_{P}$ is a valuation domain. To prove this, since $R_{P}$ is integrally closed with maximal ideal the radical of a principal ideal, it is enough to prove that for every non-zero finitely generated ideal $I$ of $R_{P}, I^{-1}$ is finitely generated [9, Exercise 39, p. 45]. This follows by observing that $I^{-1} \simeq \operatorname{Hom}_{R_{P}}\left(I, R_{P}\right)$ as $\boldsymbol{R}_{\boldsymbol{P}}$-modules and appealing to [2, Exercise 11, pp. 43-44].

Proposition 7 completely settles for integrally closed coherent domains the question of when a prime ideal is trivial: a non-zero prime ideal $P$ of a coherent normal domain $R$ is trivial if and only if $R_{P}$ is not a valuation domain; and hence all primes containing a non-zero trivial prime are also trivial. With this information at hand, Theorem 6 provides a genuine reduction of Problem II for the case " $R$ ' is integrally closed" and a true resolution of the problem in its overring formulaton for integrally closed domains $R$ :

Corollary 8. (cf. [4, Theorem 7]) Assume the notation of Theorem 6 and also assume that $R^{\prime}$ is integrally closed. Then, $(R, T)$ is coherent if and only if $\left(R, R^{\prime}\right)$ is coherent, $T$ is algebraic over $R$, and $T \subseteq R_{M}^{\prime}$ for each maximal ideal $M$ for which $R_{M}^{\prime}$ is not a valuation domain. As before, $T=\bigcap\left\{R_{P}^{\prime}: T \subseteq R_{P}^{\prime}\right\}$. 
Remark 9. As a further supplement to Theorem 6 we record some fragments concerning coherence of integral extensions. Throughout this remark $R \subsetneq T$ is assumed to be an integral extension with $R$ coherent. First note that any finitely generated intermediate $R$-algebra is also coherent [8, Corollary 1.5]. If $(R, T)$ is coherent and $T$ is finitely generated as an $R$-module, then every intermediate domain is finitely generated as an $R$-module (see proof of [15, Corollary 14]). Moreover, if $R$ is 1 -dimensional, local, and $(R, T)$ is coherent, then $R$ must be Noetherian (see Proof of [15, Theorem 15]). Finally, if $M$ is a maximal ideal of $T$, then $[T / M: R / M \cap R]$ is finite. (Proof: Replacing $R$ by $R+M$, we may assume $M \cap R=M$. Then $M$ is the conductor of an element, and hence finitely generated as an ideal of $R$. This is impossible if $[T / M: R / M]$ is not finite. Notice that the proof does not use integrality per se, but only that $M \cap R$ is maximal in $R$.

3. Prüferian questions (problem I). Observe that $\S 2$ contains a number of characterizations of Prüfer domains, all well known, except for the last one listed in the following corollary. (As usual $\bar{R}$ denotes the integral closure of the domain $R$, and let $K$ denote the quotient field of $R$.)

COROLlaRy 10. The following statements concerning the domain $R$ are equivalent:

(a) $R$ is a Prüfer domain.

(b) Each overring of $R$ is integrally closed.

(c) $R=\bar{R}$ and $(R, K)$ is INC.

(d) $R=\bar{R}$ and each overring of $R$ is of the form $\bigcap\left\{R_{P}\right\}$.

(e) $R=\bar{R}$ and each overring of $R$ is coherent.

As a special case of part (c) of Theorem 6, we then have the following corollary, due in effect to Seidenberg [18].

Corollary 11. Suppose $\operatorname{dim}(R)=1$ and $R=\bar{R}$. Then, $R$ is a Prüfer domain if and only if $R$ is coherent.

The first of the following two corollaries is immediate from Corollary 10, and the second follows directly from Corollaries 4 and 10. Let $R^{\prime}$ denote the integral closure of $R$ in a field $L$ algebraic over $R$.

Corollary 12. $(R, L)$ is INC if and only if $R^{\prime}$ (equivalently, $\bar{R}$ ) is a Prüfer domain.

Corollary 13. $(R, L)$ is coherent if and only if $\left(R, R^{\prime}\right)$ is coherent and $R^{\prime}$ is a Prüfer domain.

The requirement that $\left(R, R^{\prime}\right)$ be coherent cannot be deleted from Corollary 
13 (see Example 16(b)), but we can prove the following:

Corollary 14. If $R$ is coherent and $R^{\prime}$ is Prüfer, then each finitely generated $R$-subalgebra of $L$ is coherent.

This corollary follows immediately from Corollary 12 and the following proposition which extends Harris' theorem on the ascent of coherence along a finite integral extension of domains [8, Corollary 1.5].

Proposition 15. Let $T$ be a finitely generated $R$-algebra such that $R \subseteq T$ is an INC-extension. If $R$ is coherent, then $T$ is coherent.

Proof. Let $R^{\prime}$ denote the integral closure of $R$ in $T$. By the proof of [16, Corollaire 2] there exists a partition of unity $t_{1}, \ldots, t_{n}$ in $R^{\prime}$ such that $T_{t_{i}}$ is ring isomorphic to $\left(R_{\alpha}^{\prime}\right)_{t_{i}}$, where $R_{\alpha}^{\prime}$ is some module finite $R$-subalgebra of $R^{\prime}$ containing $t_{1}, \ldots, t_{n}$. Thus, each $T_{t_{i}}$ is coherent, since each $\left(R_{\alpha}^{\prime}\right)_{t_{i}}$ is coherent [8, Corollary 3.1]. Hence, since $T \rightarrow \Pi T_{t_{i}}$ is a faithfully flat extension [2, Proposition 2, pg. 109], we may conclude that $T$ is coherent (use [2, Corollary p. 109]).

It is appropriate at this time to mention a question of Vasconcelos and to ask a related question. He has asked if the integral closure of a 1-dimensional coherent domain is a Prüfer domain. Although no general answer is known presently, some partial solutions have been given (e.g., Gruson, in a personal communication to Vasconcelos, has proven under the above assumptions that if $R$ contains a canonical ideal, then the integral closure is a Prüfer domain. Also see $[15, \S 4]$ (Remark 9) for another partial solution.) With the above question in mind as well as Corollary 14, we raise the following question: If $\operatorname{dim}(R)=1$ with $R$ local, and if each finite type overring of $R$ is coherent, then is $\bar{R}$ a Prüfer domain?

We conclude this paper with the consideration of the following question we raised in [14, Remark 2]: If each overring of $R$ is coherent, is the poset of prime ideals of $R$ a tree (as for Prüfer domains)? The answer is "No", for [13, Example 2.28] is a counterexample. We sketch the proof below, but first we present related examples relevant to the matters under discussion above.

EXAMPLE 16. We first review the general construction that will be needed. Let $k \varsubsetneqq K$ be an algebraic extension of fields and consider a valuation domain $V$ of the form $K+M$, where $M$ is the maximal ideal of $V$. Let $R=k+M$. Then $\bar{R}=V$ and each intermediate domain of $(R, \bar{R})$ is of the form $R_{\mathrm{L}}=L+M$ for some field $k \subseteq L \subseteq K$. Observe that $M$ is finitely generated as an $R_{L}$-ideal if and only if $M$ is finitely generated as an $\bar{R}$-ideal (and hence principal) and $[K: L]$ is finite. Hence, by a direct computation or an appeal to [6, Theorem 3], we have that $R_{L}$ is coherent if and only if $M$ is a finitely generated ideal of $R_{L}$.

(a) Take $[K: k]$ finite, $\operatorname{dim} \bar{R}>1$ and $M$ principal in $V$. Then each overring of $R$ is coherent, since $(R, \bar{R})$ is coherent (Corollary 13). Furthermore $R$ is neither Prüfer nor Noetherian. 
(b) Take $M$ not to be finitely generated in $V$. Then although $\bar{R}$ is a Prüfer domain, no intermediate domain of $(R, \bar{R})$, except for $\bar{R}$, is coherent.

(c) Perform the same construction as above, except with $V$ a Prüfer domain which is the intersection of two discrete rank 2 valuation domains each of the form $K+M$, and such that $V$ has two height 1 primes (e.g., [13, Example 2.28]). Take $R=K+J$ ( $J$, the Jacobson radical of $V$ ). Then considerations similar to ones above show that $\bar{R}=V, R$ is coherent, and there are no rings properly between $R$ and $\bar{R}$. Hence, each overring of $R$ is coherent, but $R$ is 2 -dimensional and local with two height 1 primes. Whence, its poset of primes is not a tree.

ACKNOWLEDGEMENT. I am extremely grateful to the referee for his numerous improvements on an earlier version of this manuscript.

\section{REFERENCES}

1. M. F. Atiyah and I. G. MacDonald, Introduction to Commutative Algebra, Addison-Wesley, Reading, Mass., 1969.

2. N. Bourbaki, Commutative Algebra, Addison-Wesley, Reading, Mass., 1972.

3. S. U. Chase, Direct products of modules, Trans. Amer. Math. Soc., 97 (1960), 457-519.

4. E. D. Davis, Overrings of commutative rings. III: Normal Pairs, Trans. Amer. Math. Soc., 182 (1973), 175-185.

5. E. D. Davis, Integrally closed pairs, Lecture Notes in Math., Vol. 311, Springer Verlag, New York, 1970.

6. D. E. Dobbs and I. J. Papick, When is D+M coherent? Proc. Amer. Math. Soc., 56 (1976), 51-54.

7. B. Greenberg, Coherence in cartesian squares, J. of Algebra, 50 (1978), 12-25.

8. M. E. Harris, Some results on coherent rings, Proc. Amer. Math. Soc., 17 (1966), 474-479.

9. I. Kaplansky, Commutative Rings, Allyn and Bacon, Boston, Mass., 1970.

10. W. Krull, Einbettungsfreie, fast-Noethersche Ringe und ihre oberringe, Math. Nachr., 21 (1960), 319-338.

11. S. McAdam, Two conductor theorems, J. of Algebra, 23 (1972), 239-240.

12. S. McAdam, Simple going down, J. London Math. Soc. (2), 13 (1976), 167-173.

13. I. J. Papick, Topologically defined classes of going-down domains, Trans. Amer. Math. Soc., 219 (1976), 1-37.

14. I. J. Papick, A remark on coherent overrings, Can. Math. Bull., 21 (1978), 373-375.

15. I. J. Papick, Finite type extensions and coherence, Pac. J. Math., 78 (1978), 161-172.

16. M. Raynaud, Anneaux Locaux Henséliens, Lecture Notes in Math., Vol. 169, Springer Verlag, New York, 1970.

17. F. Richman, Generalized quotient rings, Proc. Amer. Math., Soc., 16 (1965), 794-799.

18. A. Seidenberg, A note on the dimension theory of rings, Pacific J. Math., 3 (1953), 505-512.

19. A. Wadsworth, Pairs of domains where all intermediate domains are Noetherian, Trans. Amer. Math. Soc., 195 (1974), 201-211.

\section{DEPARTMENT OF MATHEMATICS \\ UNIVERSITY OF MISSOURI \\ COLumbia, Missouri 65201}

